\title{
Expanding the knowledge on development of CAKUT: molecular genetics and beyond
}

\author{
Gabriel C. Dworschak ${ }^{1,2,3}$, Heiko Reutter ${ }^{3,4}$, Alina C. Hilger ${ }^{1,3}$ \\ ${ }^{1}$ Department of Pediatrics, Children's Hospital, ${ }^{2}$ Institute of Anatomy, ${ }^{3}$ Institute of Human Genetics, ${ }^{4}$ Department of Neonatology and Pediatric \\ Intensive Care, Children's Hospital, University of Bonn, Bonn, Germany \\ Correspondence to: Alina C. Hilger. Department of Pediatrics, and Institute of Human Genetics, University of Bonn, Venusberg Campus 1 , 53127 \\ Bonn, Germany. Email: alina.hilger@uni-bonn.de. \\ Provenance: This is an invited article commissioned by the Section Editor Dr. Peng Zhang (Department of Urology, Zhongnan Hospital of Wuhan \\ University, Wuhan, China). \\ Response to: Fernandez-Prado R, Kanbay M, Ortiz A, et al. Expanding congenital abnormalities of the kidney and urinary tract (CAKUT) genetics: \\ basonuclin 2 (BNC2) and lower urinary tract obstruction. Ann Transl Med 2019;7:S226.
}

Submitted Sep 20, 2019. Accepted for publication Sep 29, 2019.

doi: 10.21037/atm.2019.09.157

View this article at: http://dx.doi.org/10.21037/atm.2019.09.157

We would like to thank Dr. Fernandez-Prado, Dr. Kanbay, Dr. Ortiz, and Dr. Perez-Gomez for their insightful comments on the genetics of CAKUT (congenital abnormalities of the kidney and urinary tract), the zinc finger protein $\mathrm{BNC} 2$, and specifically on their commentary on our recent report of $B N C 2$ variants causing lower urinary tract obstruction (LUTO) (1).

In our report we showed the first monogenic cause for LUTO, identified in multiply affected families with various degrees of affection. Hence showing that developmental defects in lower urinary tract range in severity even within families carrying the same genetic variant. This follows the previously known inheritance pattern with reduced penetrance of developmental defects of the upper urinary tract and the kidneys. For isolated and non-isolated CAKUT more than 25 genes have been identified so far (2). Nevertheless only up to $10 \%$ of all affected CAKUT individuals can be identified to carry pathogenic variants in these genes (3). Up to $16 \%$ can be identified to carry a disease causing copy number variation (CNV) (2). Therefore, the genetic diagnosis of CAKUT has proven to be challenging due to genetic and phenotypic heterogeneity and incomplete genetic penetrance. In our report we also describe that only a small proportion of LUTO patients are affected due to a variant in BNC2. Overall this suggests that the pathogenesis of most CAKUT cases is multifactorial, and may be caused by polygenic and complex genetic pathways, environmental factors and epigenetics, that still need to be deciphered to gain deeper understanding of CAKUT disease mechanisms (4).

\section{Molecular function of BNC2}

Buckley et al. (5) showed that a previously associated loci at chromosome 9p22.2 conveys a risk for ovarian cancer mediated by changes in a transcriptional regulatory network. The authors established BNC2 as the most likely target gene of the risk alleles through physical DNA interactions. Using a protein binding microarray the BNC2 consensus binding sequence was determined and its enrichment was shown in BNC2 ChIP-seq, leading to a set of downstream target candidate genes. Interestingly, the analysis of the putative target genes showed enrichment of functional classes that are involved in cancer development AND embryology such as system development, anatomic structure development, multicellular organism development, and tissue development.

Hervé et al. (6) propose that $\mathrm{BNC} 1$ and $\mathrm{BNC} 2$ represent nuclear proteins specific to vertebrates. BNC1 and 2 have been shown to be the orthologs of the DISCO proteins of insects (7). All of these proteins are crucial for embryonic development. It remains to be found which targets are common to the two basonuclins and even to the DISCO proteins, and why certain cell types possess either bnc1 or 2, while others possess both. Furthermore, they have diverse functions, some of which are shared. Since BNC1 
and BNC2 comprise widely separated pairs of zinc fingers, they may bind to multiple targets, some of which may be transcribed by RNA polymerase I, and others by polymerase II (7). Yet, the targets of each pair of zinc fingers of bnc1, bnc2 and disco remain elusive. The knowledge about these targets will advance the understanding about the involvement of these proteins and how their functions have diversified in the course of evolution.

$\mathrm{Up}$ to now, mechanisms of $\mathrm{BNC} 2$ function in urinary tract development are yet speculative, since the data by Buckley et al. was acquired in ovarian cell lines. Hence, conclusions about the regulatory network and the mechanisms in embryonic tissue ultimately forming the urinary tract cannot be transferred and the effect of diseasecausing $B N C 2$ variants in LUTO patients remains elusive.

\section{Perspective}

In order to understand the role in developing urinary tract and to decipher the mechanism of the variants in $B N C 2$ experiments have to be performed in embryonic urinary tract tissue at relevant time points. Because of ethical and technical obstacles human embryonic tissues are rarely available for these experiments and scientists have to substitute with tissues or cells derived from animal models or from induced pluripotent stem cells.

The identification of $B N C 2$ as a disease gene for LUTO has improved diagnostics for clinical geneticists. However, promoter and enhancers of $B N C 2$ have not yet been screened for disease-causing variants, but the enhanced knowledge will foster understanding of non-coding variants (8). Furthermore, the screening of target genes of $B N C 2$ might identify variants in further disease-causing genes and this could help to understand the regulatory network in urinary tract development.

\section{The prospect of developing novel therapeutic approaches}

The clinical management of LUTOs is yet the management of complications and intervention may start during pregnancy as early as gestational week 13 . The first study on the effectiveness of vesicoamniotic shunting (9) has indicated a potential effect on neonatal survival rates, but evidence remains scarce. However, these interventions have not been proven to improve the renal function and this is a lingering issue in long-term morbidity and mortality. Postnatal treatment involves therapy of respiratory failure that might require ECMO therapy (10). As a consequence, therapeutic approaches preventing damage, before vesicoamniotic shunting is feasible, become increasingly interesting. As Fernandez-Prado et al. mention, the multitude of possible $B N C 2$ isoforms and the $B N C 2$ association with different diseases is complicating the use of $B N C 2$ as a therapeutic target. Yet, targeting $B N C 2$ and its' downstream network seem to be a remote objective and research has to reveal more about $\mathrm{BNC} 2$ and its' biological functions. The available animal models offer various opportunities for testing different therapeutic approaches comprising murine (11) and zebrafish models (1). These models represent a great resource for translating the basic science results into clinical practice.

\section{Acknowledgments}

None.

\section{Footnote}

Conflicts of Interest: The authors have no conflicts of interests to declare.

Ethical Statement: The authors are accountable for all aspects of the work in ensuring that questions related to the accuracy or integrity of any part of the work are appropriately investigated and resolved.

\section{References}

1. Kolvenbach CM, Dworschak GC, Frese S, et al. Rare Variants in BNC2 Are Implicated in Autosomal-Dominant Congenital Lower Urinary-Tract Obstruction. Am J Hum Genet 2019;104:994-1006.

2. Capone VP, Morello W, Taroni F, et al. Genetics of Congenital Anomalies of the Kidney and Urinary Tract: The Current State of Play. Int J Mol Sci 2017. doi: 10.3390/ijms18040796.

3. Hwang DY, Dworschak GC, Kohl S, et al. Mutations in 12 known dominant disease-causing genes clarify many congenital anomalies of the kidney and urinary tract. Kidney Int 2014;85:1429-33.

4. Nicolaou N, Renkema KY, Bongers EM, et al. Genetic, environmental, and epigenetic factors involved in CAKUT. Nat Rev Nephrol 2015;11:720-31.

5. Buckley MA, Woods NT, Tyrer JP, et al. Functional Analysis and Fine Mapping of the 9p22.2 Ovarian Cancer 
Susceptibility Locus. Cancer Res 2019;79:467-81.

6. Hervé F, Vanhoutteghem A, Djian P. Basonuclins and DISCO proteins: regulators of development in vertebrates and insects. Med Sci (Paris) 2012;28:55-61.

7. Vanhoutteghem A, Bouche C, Maciejewski-Duval A, et al. Basonuclins and disco: Orthologous zinc finger proteins essential for development in vertebrates and arthropods. Biochimie 2011;93:127-33.

8. Smedley D, Schubach M, Jacobsen JOB, et al. A WholeGenome Analysis Framework for Effective Identification of Pathogenic Regulatory Variants in Mendelian Disease. Am J Hum Genet 2016;99:595-606.

Cite this article as: Dworschak GC, Reutter H, Hilger AC. Expanding the knowledge on development of CAKUT: molecular genetics and beyond. Ann Transl Med 2019;7(20):596. doi: 10.21037/atm.2019.09.157
9. Morris RK, Malin GL, Quinlan-Jones E, et al.

Percutaneous vesicoamniotic shunting versus conservative management for fetal lower urinary tract obstruction (PLUTO): a randomised trial. Lancet 2013;382:1496-506.

10. Caesar RE, Packer MG, Kaplan GW, et al. Extracorporeal membrane oxygenation in the neonate with congenital renal disease and pulmonary hypoplasia. J Pediatr Surg 1995;30:1560-3.

11. Bhoj EJ, Ramos P, Baker LA, et al. Human balanced translocation and mouse gene inactivation implicate Basonuclin 2 in distal urethral development. Eur J Hum Genet 2011;19:540-6. 